\begin{tabular}{|c|c|c|}
\hline & $\begin{array}{l}\text { European Association for the } \\
\text { Development of Renewable Energies, Environment } \\
\text { and Power Quality (EA4EPQ) }\end{array}$ & $\begin{array}{l}\text { International Conference on Renewable Energies and Power Quality } \\
\text { (ICREPQ'12) } \\
\text { Santiago de Compostela (Spain), 28th to 30th March, } 2012\end{array}$ \\
\hline
\end{tabular}

\title{
Fluent Restoration Modelling Applied to a Real Power System Using Colored Petri Nets
}

\author{
K.M. Canuto ${ }^{1}$, G.C. Barroso ${ }^{2}$, M.P. Sales Neto ${ }^{1}$, R.F. Sampaio ${ }^{2}$, R.P.S. Leão ${ }^{2}$ and E.B. Medeiros ${ }^{1}$ \\ ${ }^{1}$ Energy Company of Ceará - COELCE \\ Rua Pe Valdevino, 150 Joaquim Távora - Fortaleza-CE (Brazil) \\ E-mail: klenson@coelce.com.br,moriton@coelce.com.br,eudes@coelce.com.br \\ ${ }^{2}$ Federal University of Ceará \\ Caixa Postal 6001 - Campus do Pici - CEP: 60455-760 - Fortaleza-CE (Brazil) \\ Phone: +55 85 3366.9576, fax: +55 85 3366.9574, e-mail: gcb@fisica.ufc.br, rfurtado@dee.ufc.br, rleao@dee.ufc.br
}

\begin{abstract}
In this article the Colored Petri Nets (CPN) and the Critical Path Method along with the Program Evaluation Review Technique (CPM/PERT) are described and their characteristics compared for application on the real-time fluent restoration of power systems. A CPN based method is proposed and has been found more suitable since it is able to represent the temporal sequencing and the logical dependency between restoration activities. The restoration planning of electrical power systems are based on a worksheet drawn up by experts, which contains a list of procedures for restoration. The restoration process has been improved and a real case study is presented using the CPN modeling applied with success to an operative fluent restoration post-outage in the Brazilian interconnected system.
\end{abstract}

\section{Key words}

Fluent restoration, Colored Petri nets, CPM/PERT, Brazilian interconnected system.

\section{Introduction}

When planning the restoration of power systems, it is highly desirable to develop a computational approach to represent restoration plans. The approach should deal suitably with the problems inherent to the activities of power system reenergizing, according to a logicalmathematical procedure.

When choosing the approach, it must be taken into consideration its ability to represent the different nature of the numerous tasks that are performed during the process of restoration. The activities can differ in nature such as: technical, as for instance, the control actions, monitoring the operational restrictions of the system components; management, as for example, the coordination of interconnection of different geo-electric areas; logistics, such as the displacement of maintenance teams and transport cars; and strategic, as representing the priority for reenergizing loads [1]. Furthermore, two very important aspects must be taken into account when defining a model for restoration plans, which are: a) the possibility to qualify the different lines of action set out to tackle the reenergizing process under a given scenario of an outage, covering the different aspects of the process; b) the viability to model the imprecise nature of the activities of restoration, allowing a stochastic approach in its specification.

Usually restoration plans are textual procedures of the restoration activities, and there are drawbacks of the textual form as pointed out in [2]. A lot of efforts have been made including visualization and expert system applications.

This paper presents a study of two methods, CPM/PERT and Colored Petri Nets (CPN), both suitable for computer implementation of restoration strategies of power systems under outage conditions. A modelling using CPN has been developed and applied to a real disturbance occurred in a $69 \mathrm{kV}$ network of the Company of Energy of Ceará - Coelce, in Brazil. The approach was based on the Operative Statement (OS) for fluent load transfer among ten areas, based on study of loading, voltage level and protection sensitivity of the power system areas.

\section{Methods of Restoration of Power Systems}

The basic characteristics of the computational models $\mathrm{CPM} / \mathrm{PERT}$ and CPN employed in power system restoration plans are examined next.

\section{A. CPM/PERT Methods}

The CPM/PERT are techniques for scheduling a set of activities, developed during the $1950 \mathrm{~s}$ by the United States Navy and the DuPont Company. The goal of $\mathrm{CPM} / \mathrm{PERT}$ methods is to optimize processes with 
multiple chained activities, both in the temporal aspect as well as in the application of resources [3][4]. These methods define a formal basis and a generalised approach to projects management. In addition, they deal with features of flexibility and imprecisions, normally found in plans of restoration. Both techniques assume that any project or plan can be broken down into a logical sequence of predefined chained actions (or activities). Carrying out these actions make possible the project completion. Another important feature of these methods is that the activities have technological implementation logic, i.e., although they can be implemented in a manner relatively independent, they have necessarily technological precedence among themselves. In its formulation, it is assumed that the relationship between duration and costs of each task in the process is well known. This allows the estimation of various management and performance related parameters, optimal allocation of resources and control of project costs.

CPM/PERT methods are based on identifying the critical path of a process, that is, the sequence of activities that determine the total duration of the project. To achieve this goal, both methods use a graphical representation of the relationships between process activities, in the form of a graph-oriented named graph CPM/PERT.

Thus, the approach of a problem through methods of critical path is performed from the initial definition of the list of process activity under analysis. By setting the list of activities, the CPM/PERT graph can be built to facilitate the implementation of the algorithms, and calculate efficiently the costs and time involved in the project.

Activities are represented by oriented arcs, which begin and end in discrete steps. These arcs are related to different characteristics such as time estimation for conclusion of each activity, resources involved and the relationship between cost and time in the process. Using an approach based on objects, instancing activities of a plan can be performed directly from the list of activities associated with the technological process in question.

\section{B. Petri Nets Method}

The Petri nets (PN) are mathematical and graphical method that can model discrete event systems. The method makes easy to model situations that hardly are represented by other templates, such as concurrency, parallelism, synchronization between multiple processes, competition for a resource, etc., what makes it a very important tool. The PN are most appropriate to represent formal parallelism and synchronization in engineering environments. Therefore, the use of PN to the modelling, analysis and control of discrete events are receiving greater attention in recent years for various reasons as listed next $[5]$.

The PN has graphical format easy to understand, where complex systems can be represented. It is able of modelling concurrent and asynchronous events, relations of logical precedence and structural interactions in a way simple and natural. Conflicts, deadlocks, finite buffers, synchronizations, priorities, and operations of mounting and dismounting can be modelled easily and efficiently.

The PN can also be applied to implement real-time systems [6] and it is successfully applied to power system failures [7].

\section{Restoration Plans}

The Grid Code in Brazil presents provisions for the Interconnected National System (SIN) when subjected to outage, which are termed Operational Instructions (OI) for restoration. An OI is a document that describes the actions that must be taken and the conditions that must be fulfilled in the operation of SIN, after an outage of major proportion takes place.

The restoration procedure of the Brazilian power system is organized in two stages according to the national system operator (ONS): fluent stage and coordinated stage [8]. The fluent stage corresponds to the beginning of the process of restoration, where it is supposed that the system is de-energized. This stage requires operators to take decisions following a set of instructions found in the manuals of operation, with minimal communication between the operators involved. The coordinated phase encompasses the connection of the areas already restored during the fluent restoration [9].

Figure 1 shows an example of fluent transfer between three areas to a configuration with the supply loss in area A. In this case, the circuit breaker that connects the areas $\mathrm{A}$ and $\mathrm{B}$ is switched on, causing the Area B take on the loads of Area A.

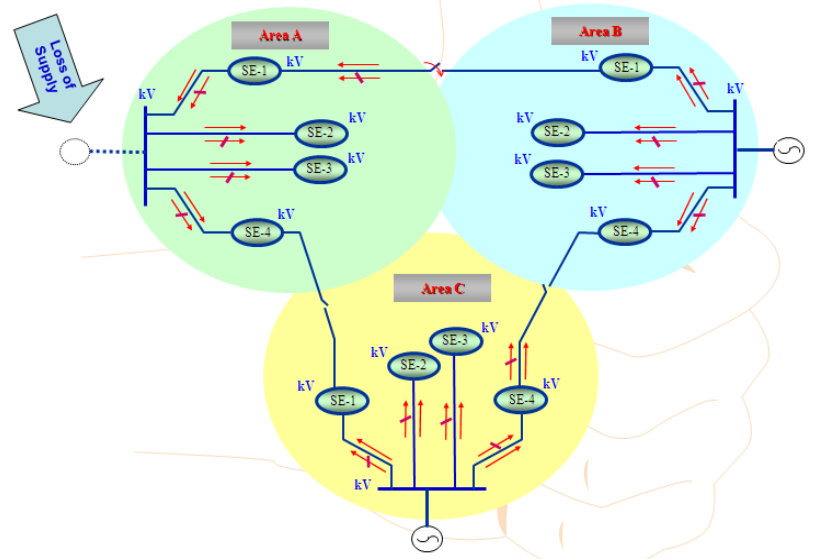

Fig. 1. Example of fluent transfer procedure in a power system with three control areas: A, B and C.

\section{Tabular Format of Restoration Plans}

The system operator in Brazil recommends the representation of the OI in a tabular format for easy understanding by operators and engineers of the activities described in the restoration plan. This format makes explicit the important characteristics of the process, such as the order of a particular activity following the planned actions, the performer of this activity, the specific 
procedures and conditions (or controls) that must be met before an action takes place.

This paper presents a computer approach to modelling the fluent restoration plan of the $69 \mathrm{kV}$ network of the Coelce Company. The network has ten different points of connection with the SIN, which are supplied by the generation and transmission utility Sao Francisco Hydroelectric Company - Chesf.

\section{Comparison between CPM/PERT and Petri Nets}

A CPM/PERT graph is a graphical representation of the relationship between various activities, representing operations that consume time and resources. CPM/PERT graphs have a single initial node and a single end node, which indicates that any path followed starting from the initial event, always lead to the final event. Thus, the longest path is called the critical path, which corresponds to the sequence of activities that must exercise control on it. Any delay in critical path automatically determines a delay in the project.

There are two ways to represent a CPM/PERT network [8]. Figure 2 shows the representation of activities by arcs (A-on-A) or the American method. The occurrence of the event 5, for example, means that the activities "e" and " $f$ " were completed and the activity "g" can only be started if and only if " $\mathrm{e}$ " and " $\mathrm{f}$ " were completed. In some cases it is necessary to use ghosts activities to represent certain constraint relationships between nodes.

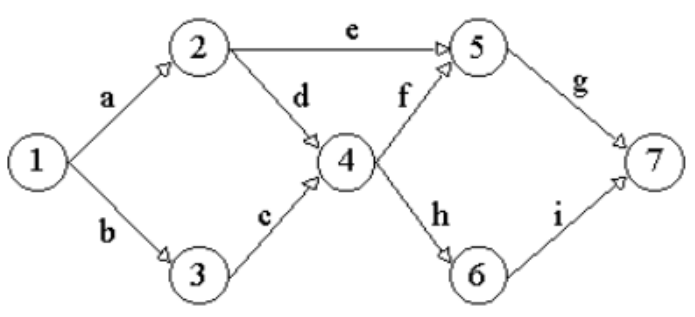

Fig. 2. CPM/PERT graph A-on-A type, in which arcs represent activities and nodes represent logical events.

Figure 3 shows the representation of activities by nodes (A-on-N) or French method. The network in Figure 3 represents the same CPM/PERT network of Figure 2 by Aon-N method. An important feature is that this type of network is not required fictitious activities, except for initial and final nodes.

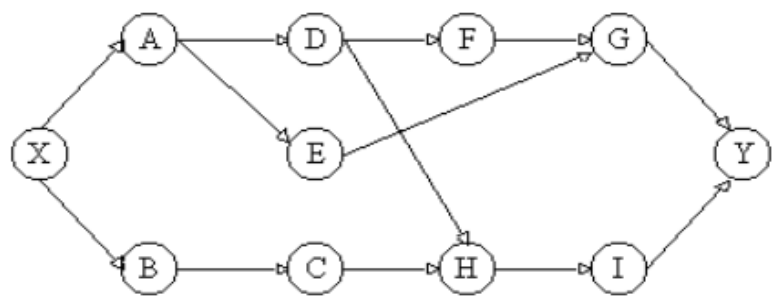

Fig. 3. CPM/PERT graph A-on-N type, where nodes represent activities and arcs represent relations of precedence between the activities.
The main use of CPM/PERT networks is the determination of the duration of a project using the method of critical path. The basic difference between PERT and CPM method is that the first estimates the duration for each project task or operation, relying simply on cost information; whilst the second method deals with duration and cost, which derives from a number of durations for each activity. Choosing the appropriate duration causes the project total cost to be minimal.

Since a CPM/PERT graph consists of activities and events, it can easily be converted into a PN net. Thus, in a CPM/PERT network A-on-A type, each activity can associate a transition, and each event a place (double). On the other hand, in a PN net each transition must be preceded by a place that represents a different condition for each transition. Figure 4 represents the PN net corresponding the networks shown in figures 2 and 3.

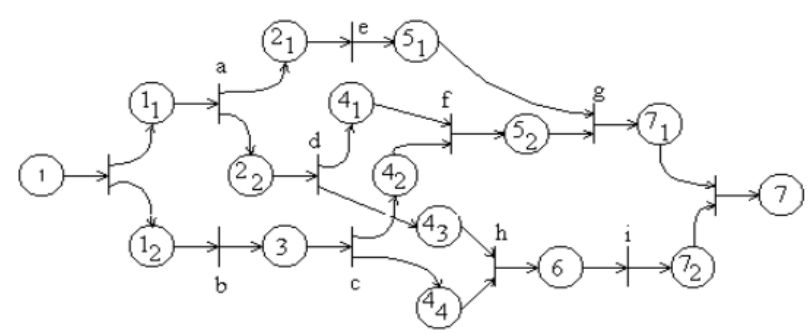

Fig. 4. PN graph corresponding to CPM/PERT graphs of figures 2 and 3.

The PN are therefore an excellent approach for representing relations of precedence and competition also represented by CPM/PERT networks. The determination of the critical path of operations is of particular importance. The critical path restricts the overall project program in the sense that the transactions that are part of the critical path have no slack. Therefore, any delay time in any of these operations puts in danger the runtime of the project.

In the case of PN, circuits can be used to study the behaviour of the trigger limiting. Circuits with a maximum period of time are called critical circuits. Therefore, the operations associated with these circuits may restrict the implementation of the system in the sense that, if the duration of any critical circuit operation is incremented, the duration of the cycle of the system also will be.

As in the CPM/PERT in which critical path can change when the duration of certain activities changes (activities in the critical path), the PN critical circuits may also change when the durations of the operations are changed. Therefore, an increase in the duration of an operation may only increase marginally the cycle time of the system. In this sense, the concept of criticality in the PN is also of vital importance.

Some other advantages of the PN method on PERT/CPM methods are as follows: The PN admit repetitive cycles, while CPM/CPM networks cycles are not supported; resource requirements for the implementation of activities appears explicitly in the $\mathrm{PN}$ representation 
through tokens, and the model CPM/PERT cannot describe the non-determinism, such as: selection and repetition which are critical properties of a restoration plan.

Changes in a model of PN are made simply by adding tokens, places or transitions. On the other hand, changes in the model CPM/PERT usually require a redefinition of all states in the model. The PN is a model for easy learning, and work as language of communication between specialists in different areas.

In the next section will be shown a case study of fluent restoration, where the PN method has been applied for the benefits explained.

\section{Modelling of Restoration of Power Systems using Colored Petri Nets: Case Study}

Until recently, upon the loss of significant part of the electrical system there was need for analysis and approval of ONS to recompose the load affected by other supply point. The restoration was only authorised by ONS after the completion of the methodological analysis of the amount of load to be transferred, as well as the predicted loading in power transformers localized in the recipient point of supply. This procedure caused delay in the restoration of the power system and an increase in customer dissatisfaction.

The Technical Operation team of Coelce has conducted study that culminated in the elaboration of procedures for fluent transfer of load between control areas without compromising the national interconnected grid performance. The study has considered the loading of transformers of the transmission utility - Chesf.

During the study was applied the concept of fluent restoration without prior authorization by ONS recommended in the Brazilian grid code [10].

The OI for fluent restoration among areas was based on the evaluation of transformers loading, voltage level and coordination of protection of the areas.

The accomplished study was submitted for analysis by ONS, which culminated in the prior approval granted to Coelce utility. Based on the study proposal, ONS considered that the collaborators of the power distribution utility had demonstrated domain in matters related to fluent transfer procedures. The study has considered assumptions as outlined next.

Initially it was prepared a worksheet with information taking into account the following assumptions:

- Mapping of loads that will be affected;

- $\quad$ Loading of the supplier axis in normal condition (N) and in condition of contingency $(\mathrm{N}-1)$;

- Loading of power transformers of the transmission utility in N-1 condition up to $120 \%$;

- $\quad$ Pick-up and sensitivity of relay protections;

- Voltage levels;
- $\quad$ An update of the study and submission to ONS at each four-months.

The worksheet was divided in various groups of information as: source area, destination area, rated power of transformers of the transmission utility, decision to be taken and operational restrictions. The worksheet reports a total of 23 transfer procedures between areas, stating for each transfer the destination area, substations to be transferred and the amount of load in MVA. The study takes into consideration the three load scenarios: heavy, medium and light load condition, as well whether the destination area has all power transformers installed in the area $(\mathrm{N}$ or $\mathrm{N}-1)$ available. The last check is whether the power transformers of the destination area support an overload of up to $120 \%$ for a maximum period of 30 minutes.

Based on the datasheet developed by experts, a Fluent Load Restoration System (FRS) was developed for the electrical system of Coelce. The FRS aids the system operator in decision-making in the Control Centre of Coelce. The design of the FRS was based on CPN method, using the CPN Tools [11], a tool for editing, simulating and analysing Colored Petri nets, which efficiently handles untimed and timed nets. The output of the CPN model provides information to the system operator, stating whether the transfer is possible.

The FRS was programmed according to the worksheet approved by ONS. The worksheet was elaborated for each four months period of the year, taking into consideration the growth of the energy market by 2012 . The FRS is able to make the task of fluent restoration less stressful, independent of emotional conditions of the system operator and provide the operator an accurate and succinct diagnosis about the system.

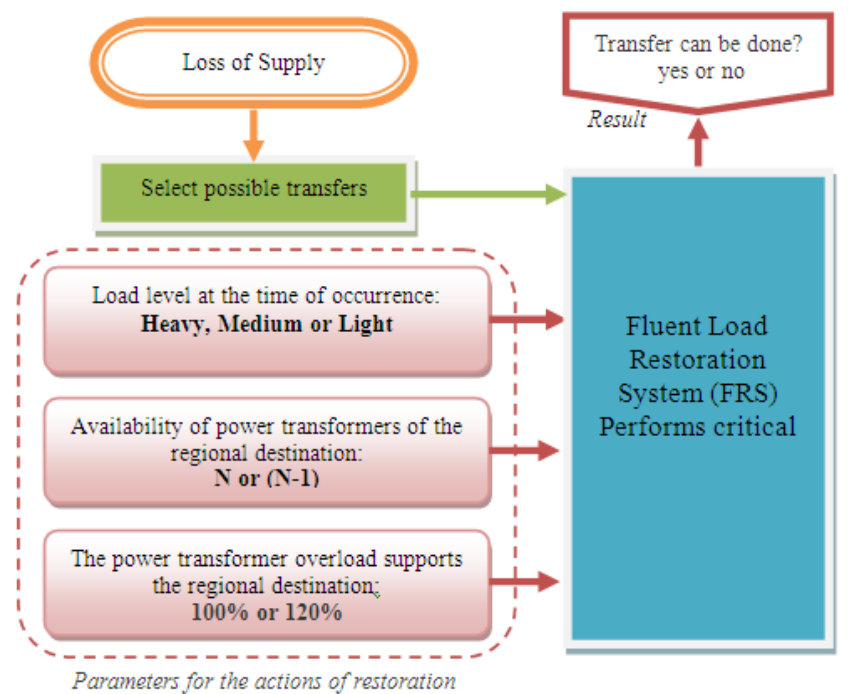

Fig. 5. Flowchart of the Fluent Load Restoration System process.

The FRS starts to run when the power system undergoes a loss of power supply in a control area. The faulty section is isolated and then the new system condition updated. The next step is to check the possibility of 
restoration of the affected loads and test if the power supply in the destination area is normal as depicted in Figure 5. The FRS identifies the substations and the parts of the system that will be transferred, identifying the source and destination areas.

The FRS can also be used as a tool for system operators' training, which can be run in random mode; the system randomly chooses a load transfer to be analysed.

\section{A. CPN Main Page of the FRS}

Figure 6 shows the flowchart of the main page of the model depicted in software CPN Tools. On that first page are the tokens that identify the 23 possible transfers and the types of variables. After all enabled transitions are fired, the token will identify whether the chosen transfer is suitable or not, taking into account the three main variables: power transformer loading, voltage level and protection sensitivity.

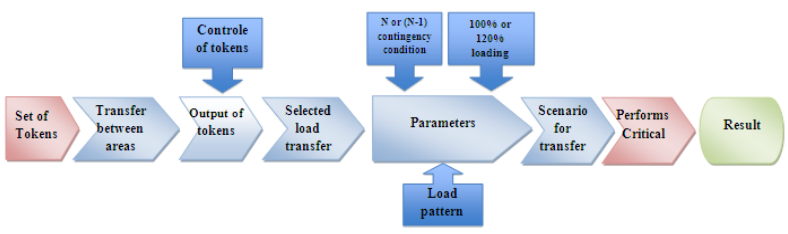

Fig. 6. Flowchart of the CPN main page of the FRS model.

In the 23 enrolled tokens of transfer are given the "origin area/destination area". The tokens that make the initial marking are in place "transfer between areas". Information about the chosen transfer along with information obtained through the combination of the three variables will result in the final diagnosis.

Another advantage of FRS is the ease of updating the network when new transfers or change of variables of target area are inserted. To update the CPN model, it is need just update the tokens in place "transfer between area", i.e., to register a new transfer and change the programming on the arc corresponding to new transfer as specified at the worksheet. It is worthy to note that the structure of the model of the network does not change.

The place "Selected Load Transfer" shows the transfer that will be examined by CPN network, getting also token 1' (n, p) type. The transition "Parameters", which is a replacement transition linked to the Sub-page "PARAMETERS", is supported by tokens with formatting $1^{\prime}(\mathrm{n}, \mathrm{p}, \mathrm{y}, \mathrm{z}, \mathrm{x})$, where:

- "n" represents the number of transfer

- " "p" represents the from/to areas;

- "y" represents if the destination area has all the power transformers available indicated by "N-1" or some transformer is unavailable, i.e., in maintenance, in this case indicated by "N";

- " $x$ " represents if at the time the transfer, the destination area is in one of the following load pattern: heavy, medium or light; indicated respectively as: "PE", "ME" and "LE";
- " $z$ " represents if the transformers at destination area can overload up to $120 \%$ for a maximum period of 30 minutes or not, indicated respectively as: "120\%" and "100\%".

An example is given for the token format: 1 ( 1 ,"MLG/TAD","N","ME","100\%"), where MLG is the origin area, TAD is the destination area, $\mathrm{N}$ is the contingency condition, ME is the load pattern and $100 \%$ is the transformer loading condition.

\section{B. Sub-Page Parameters}

Figure 7 shows the Sub-page 'PARAMETERS', where the token type $1^{\prime}(n, p, y, z, x)$ reaches transition "Parameters", making it ready to fire according to the programme in the 20 arcs that connects transition "Parameter" to the place "Result". Each arc outgoing transition "Parameters" has a specific program that leads to an indication of a token type "YES" in case of a condition imposed on the arc is fulfilled. Otherwise, the arcs will not mark tokens in place "Results".

The FRS shows to the operator of the system if the chosen transfer option is suitable or not. An example of a likely output is:

if $((\mathrm{p}=" \mathrm{BNB} / \mathrm{RSD}$ " or else $\mathrm{p}=$ "RSD/BNB" or else $\mathrm{p}=$ "SBD/CPE") and also $\mathrm{y}=" \mathrm{~N}-1$ " and also $\mathrm{z}=" 120 \% "$ and also $\mathrm{x}=$ "ME") then 1 "YES"

else empty.

The instruction meaning is: $p=$ "origin area/destination area" for $\mathrm{BNB} / \mathrm{RSD}$ or else $\mathrm{RSD} / \mathrm{BNB}$ or else $\mathrm{SBD} / \mathrm{CPE}$; $y=" N-1 "$ means the contingency state; $z=" 120 \% "$ is the loading that the power transformer in the destination area can take up during $30 \mathrm{~min}$; and $\mathrm{x}=$ "ME" is the medium load pattern. If all these conditions are fulfilled then the transfer is possible. Otherwise, it is not.

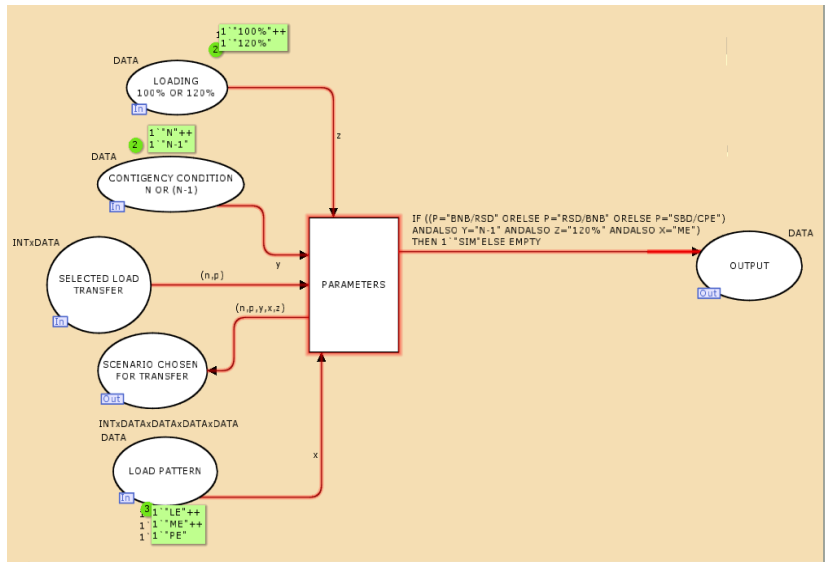

Fig. 7. Sub-page Parameters of the FRS model.

\section{Conclusions}

The methods CPM/PERT and CPN can be applied for modelling the restoration of electrical power systems. Based on the characteristics of the two approaches, the CPN method was chosen for designing a system for Fluent Restoration (FRS) for a sub-transmission network of $69 \mathrm{kV}$ of the Energy Company of Ceará in Brazil. The 
developed system, however, can be applied to any electrical power system without changing the structure of the CPN network. The response of the proposed SFR is fast and accurate, and updates in the power system are easily implemented in the model just by the addition of new tokens and arcs (if necessary).

The developed FRS gives more autonomy to the Control Centre, reduces customer downtime and improves customer satisfaction and the indices of quality of service of the company.

The tool offers a training environment for power system operators and overall it is a valuable resource to aid in the decision-making of system operators in emergency situations.

With the results achieved, the national system operator (ONS) has recommended the same procedure deployed by the Coelce to the other power distribution utilities in the country.

\section{Acknowledgement}

The authors acknowledge COELCE for the support to this work.

\section{References}

[1] M. M. Adibi, "Power System Restoration - Methodologies and Implementation Strategies", New York: IEEE Press 2000.

[2] A. A. Mota L. T. M. Mota, and A. Morelato, "Visualization of Power Systems Restoration Using CPM/PERT Graphs", IEEE Trans. On Power Systems, Vol. 22, No 3, pp.1322-1329, August 2007.

[3] J. J. Moder and C. R. Phillips " Project Management with CPM and Pert" New York: Van Nostrand Reinhold Company 1970.

[4] B. D. Sivazlian and L. E. Stanfel, "Optimization Techniques in Operations Research” New Jersey: PrenticeHall 1975.

[5] K. H. Lee, J. Favrel and P. Baptiste, "Generalized Petri Net Reduction Method". IEEE Transaction on System man and Cybernetics, vol. SMC-17, n- 2, pp. 297-303, March/April 1987.

[6] P. Gomes, A. P. Guarini, A. C. S. de Lima, M. C. Guarini, "Guidelines for Power System Restoration in the Brazilian System", IEEE Transactions on Power Systems, vol. 19, no2, pp.1159-1164, May 2004.

[7] C. L. Yang and J. Li, "Visualization and Support of Power System Restoration Using Hierarchical Color Petri Net", IEEE Canadian Conference on Electrical and Computer Engineering, pp. 439-442, May 2008.

[8] J. A. Grislain and L. Pun, "Graphical Methods for Production Control". Int. J. Pro. Res., vol. 17, n. 6, pp. 643-659, 1979.

[9] N. Martins, E. J. Oliveira, W. C. Moreira, J. L. R. Pereira e R. M. Fontoura, "Redispatch to Reduce Rotor Shaft Impacts Upon Transmission Loop Closure" IEEE Transactions on Power. Vol. 1, no.1, pp. 1-8, 2006.

[10] www.ons.org.br, accessed in 13/03/2011.

[11] http://cpntools.org/, accessed in 28/04/2011. 